\title{
Método Análisis Envolvente de Datos y Redes Neuronales en la Evaluación y Predicción de la Eficiencia Técnica de Pequeñas Empresas Exportadoras
}

\author{
Tomás Fontalvo(1), Efraín De La Hoz ${ }^{(1)^{*}}$ y Enrique De La Hoz ${ }^{(2)}$ \\ (1) Universidad de Cartagena, Facultad de Ciencias Económicas, Programa Administración Industrial. \\ Campus Piedra de Bolívar, Cartagena - Colombia. (e-mail: tfontalvoh@unicartagena.edu.co; \\ edelahozg@unicartagena.edu.co). \\ (2) Universidad Tecnológica de Bolívar, Facultad de Ingeniería, Programa de Ingeniería Industria. Campus \\ Ternera, Cartagena - Colombia. (e-mail: edelahoz@utb.edu.co).
}

*Autor a quien debe ser dirigida la correspondencia

Recibido Mar. 6, 2018; Aceptado May. 15, 2018; Versión final Jul. 23, 2018, Publicado Dic. 2018

\begin{abstract}
Resumen
En esta investigación, se desarrolló un método para evaluar y predecir la eficiencia de pequeñas empresas exportadoras tomando como variables de entrada o recurso los rubros financieros total activos, patrimonio, total pasivo, gastos operacionales, costos de ventas y como variables de salida o resultado las ventas netas, utilidad neta y utilidad operacional. Para esto se utilizó el análisis envolvente de datos en la evaluación de la eficiencia, el análisis discriminante en la valoración de la clasificación de empresas eficientes y no eficientes y las redes neuronales artificiales para evaluar su capacidad de predicción clasificatoria en 90 empresas del sector de la ciudad de Barranquilla-Colombia. Los resultados permitieron clasificar las empresas según nivel de eficiencia mostrándose una eficiencia técnica promedio del $41,38 \%$ del sector con 11 empresas representativas de la eficiencia. Los resultados muestran la relevancia de la metodología propuesta para clasificar y pronosticar correctamente la eficiencia técnica en las pequeñas empresas exportadoras.
\end{abstract}

\section{Data Envelopment Analysis Method and Neural Networks in the Evaluation and Prediction of the Technical Efficiency of Small Exporting Companies}

\begin{abstract}
In this research, a method was developed to evaluate and predict the efficiency of small exporting companies taking as input or asset variables the total assets, equity, total liabilities, operating expenses, sales costs and as output or result variables. net sales, net income and operating income. For this, the envelopment data analysis was used in the evaluation of the efficiency, the discriminant analysis in the evaluation of the classification of efficient and inefficient companies and the artificial neural networks to evaluate its capacity of classification prediction in 90 companies of the sector of the city of Barranquilla-Colombia. The results allowed to classify the companies according to level of efficiency showing an average technical efficiency of $41.38 \%$ of the sector with 11 representative companies of efficiency. The results show the relevance of the proposed methodology to correctly classify and forecast technical efficiency in small exporting companies.
\end{abstract}

Keywords: technical efficiency; data envelopment analysis; artificial neural networks 


\section{INTRODUCCIÓN}

Los mercados globalizados proponen exigencias en las condiciones competitivas del sector empresarial, en especial las empresas inmersas en la dinámica del comercio internacional. Bajo estas condiciones es necesario que se estudien las estructuras operativas y financieras de las organizaciones que exportan para analizar las variables y factores financieros y tomar decisiones que contribuyan con su competitividad empresarial. Desde esta perspectiva, se hace necesario el estudio de la eficiencia técnica de las variables y factores que inciden en su mejora. Así como el análisis y estructuración de modelos que permitan pronosticar la eficiencia de una empresa. Lo anterior genera un espacio de análisis y reflexión que le da sentido a las preguntas problemas de esta investigación.

¿Cómo determinar las variables que permitan evaluar la eficiencia técnica de las pequeñas empresas exportadoras de la ciudad de Barranquilla?, ¿Cómo valorar la eficiencia de las pequeñas empresas exportadoras de la ciudad de Barranquilla?, ¿Cómo establecer una función o modelo matemático que permita predecir la pertenencia de las pequeñas empresas exportadoras eficientes y no eficientes de la ciudad de Barranquilla?, ¿Cómo pronosticar la eficiencia o no de nuevas pequeñas empresas exportadoras de la ciudad de Barranquilla?, ¿Cómo validar el modelo seleccionado, a través de cálculo multivariado para predecir la pertenencia de pequeñas empresas certificadas y no certificadas de la ciudad de Barranquilla?, ¿Cuáles son las variables de mayor peso para predecir la eficiencia o no de pequeñas empresas exportadoras de la ciudad de Barranquilla? Este conjunto de interrogantes generó la necesidad del planteamiento del objetivo de esta investigación el cual fue establecer una método de evaluación y predicción de la eficiencia técnica de las pequeñas empresas exportadoras de la ciudad de Barranquilla a través de la utilización de herramientas de análisis envolvente de datos y las técnicas de cálculo multivariado, redes neuronales y análisis discriminante.

El Análisis Envolvente de Datos AED es un modelo que tiene como propósito evaluar diferentes tipos de eficiencia a un grupo de empresas o unidades de toma de decisiones que tienen un propósito común. EI AED también llamado análisis de frontera, constituye una herramienta de gestión utilizada en el cálculo de la eficiencia comparada de las unidades de producción (Serna, et al, 2007). Otros autores han desarrollado la técnica en diversos contextos como la evaluación de desempeño (Lins, et al, 2007), la evaluación eficiencia económica (Heidari, et al, 2011), la eficiencia gerencial (Tsay, et al, 2017), así como la evaluación de esquemas optimizados (Zhou, et al, 2015). Otras investigaciones han utilizado AED para el análisis de eficiencia multidimensional (Soares, et al, 2017) así como los procesos de internacionalización de mercados (Cano, et al, 2017).Matemáticamente podemos aseverar que El Análisis Envolvente de Datos es un modelo de optimización consistente en una función objetivo $\mathrm{h}_{0}$ (Ecuación 1) que representa un índice de eficiencia, $\mathrm{y}$ un conjunto de restricciones conformadas por ecuaciones y/o inecuaciones que expresan condiciones limitantes para el sistema (Ecuación 2). Para lo anterior la función objetivo se establece mediante el cociente de las variables de salida o resultados y las variables de entrada o recursos de las empresas o entidades objeto de estudio.

EI Modelo DEA CCR por las siglas de los autores Charnes, Cooper y Rhodes también definido como eficiencia técnica, y modelos con retornos a escala constantes, se define como la relación de la sumatoria ponderada de las salidas sobre la suma ponderada de las entradas. La intencionalidad del modelo CCR es maximizar la eficiencia de una empresa o unidad de medida de decisión DMU, dentro de un grupo de organizaciones de referencias, mediante los pesos óptimos relacionados a las variables de entrada y salida (Fontalvo, et al, 2015). Como modelo complementario también existe el modelo BCC de eficiencia pura o administrativa 0 modelos conocidos también como modelo con retornos a escala constante. La diferencia entre el modelo CCR y el modelo BCC Banker, Charnes y Cooper. Radica en la restricción de convexidad requerida por el modelo que mide la eficiencia pura o administrativa. (Guzmán, 2005)

El proceso de optimización comprende determinar endógenamente los pesos de los criterios de rendimiento (Ahn, et al, 2012) y los valores de las variables para lograr el valor máximo o mínimo de la función objetivo (García, 2009). El análisis DEA es una técnica no paramétrica que determina una frontera de eficiencia relativa a partir del tratamiento de varias variables de entrada (recursos) y varias variables de salida (productos 0 resultados) (Visbal-Cadavid et al, 2015).

$$
\begin{aligned}
& \operatorname{maxh}_{o}=\frac{\sum_{r=1}^{s} U_{r} * y_{r o}}{\sum_{i=1}^{m} V_{i} * x_{i o}} \\
& \text { Sujeto a: } \frac{\sum_{r=1}^{s} U_{r} * y_{r j}}{\sum_{i=1}^{m} V_{i} * x_{i j}} \leq \text { ó } \geq \text { ó }=1 \quad j=1, \ldots, n
\end{aligned}
$$

Con $h_{o}$ el índice de eficiencia de la unidad observada o, s número de variables de salida o resultado, m número de variables de entrada o recurso, $U_{r}$ peso relativo de la variable r-ésima de salida o resultado (positiva y desconocida), $y_{r o}$ cantidad de la variable de salida r-ésima en la observación $0, V_{i}$ peso relativo de la variable 
i-ésima de entrada o recurso (positiva y desconocida), $x_{i o}$ cantidad de la variable de salida i-ésima en la observación o y n cantidad de observaciones analizadas. El análisis DEA se fundamenta en el análisis microeconómico estándar, la econometría y la programación matemática para identificar explícitamente una frontera eficiente para determinar una medida global de eficiencia que permite ordenar las organizaciones (García, 2009). Otras investigaciones también han abordado este tipo de estudios en otros contextos empresariales como se puede observar en los resultados de investigaciones (Hanafizadeh, et al, 2014) donde también se utilizan modelos neuro DEA para medir la eficiencia en fondos de inversión. Por otro lado otros estudios (Mojtaba G, in press) analizan modelos DEA utilizando entradas controladas y no controladas en los procesos de optimización. Así mismo, al igual que en esta investigación estudios similares articulan dos enfoques técnicos y trabajan con análisis difuso y los modelos DEA (Khalili-Damghani y MohammadTaghavifard, 2012).

Es importante señalar que trabajos como los de Quesada (2003), Kwak, et al (2009), García (2009), Delfín y Melo (2017) y Moreno, López y Díaz (2014) muestran la utilidad del análisis envolvente de datos para evaluar la eficiencia de las organizaciones a partir de múltiples entradas (recursos) y salidas, pero en esta investigación, adicionalmente se hace una integración metodológica de la herramienta DEA y la RNA para valorar y pronosticar la eficiencia técnica de empresas, con lo cual se hace un aporte al desarrollo de metodologías de análisis de las organizaciones.

Por otro lado, otros investigadores han abordado el estudio de las redes neuronales artificiales. Según Bishop (2008), las redes neuronales artificiales (RNA) es una técnica computacional basada en el proceso de aprendizaje, con amplia y gran potencial de aplicación (Alaka, et al, 2017). Se basan en las características funcionales del sistema nervioso humano, utiliza un modelo de optimización no lineal (Miranda y Guzmán, 2017). Por su parte, McMillan (2013) conceptúa las RNA como redes compuestas de nodos de cómputo no lineal que incorporan una aproximación a las neuronas biológicas. Altamirano et al (2017) plantean que las RNA son estructuras capaces de imitar las funciones del cerebro humano para aprender relaciones complejas de datos, para identificar patrones y hacer predicciones acerca de nuevos datos, pueden usarse para determinar la pertenencia de un elemento desconocido a una categoría o para establecer un valor numérico especifico; su modelamiento implica la preparación de los datos, el entrenamiento y prueba de la red y la predicción de resultados. Otros autores como Hornik, et al (1989); Jiahe, et al (2003); Martín, et al (2007); Reddy (2005); Velásquez, et al (2009) lo asocian a un modelo matemático que imita de manera simplificada el procesamiento de información del cerebro. Matemáticamente, la sumatoria de los diferentes valores modificados por los pesos sinápticos, que determinan si la neurona se activa o no, se calcula mediante la ecuación 1.

$$
\mathrm{Net}_{\mathrm{j}}=\sum_{\mathrm{i}=1}^{\mathrm{N}} \mathrm{x}_{\mathrm{i}} \mathrm{w}_{\mathrm{ji}}+\theta_{\mathrm{j}}
$$

Donde $N e t_{j}$ representa la entrada neta, $w_{j i}$ el peso sináptico de la neurona $j$ sobre la entrada $x_{i}$. La neurona se activa mediante una función de activación, la cual propaga la salida $y_{j}$ de la neurona hacia otra neurona o la salida de la red como se muestra en la ecuación 2 (Caicedo y López, 2009).

$$
\mathrm{y}_{\mathrm{j}}=\operatorname{Fact}\left(\mathrm{Net}_{\mathrm{j}}\right)
$$

En esta investigación se utiliza el perceptrón multicapa, el cual según Hernández et al. (2005) construye una red en forma de cascada con una o más capas ocultas.

\section{METOdOLOGÍA}

Para el desarrollo de esta investigación se tomaron las variables preliminares, teniendo en cuenta la información disponible y consistente aportada por la Cámara de comercio de Barranquilla, asociada a los rubros financieros de las pequeñas empresas exportadoras de Barranquilla. Considerando lo anterior los investigadores a través de un análisis racional estructuraron los rubros del modelo AED que se constituyen en insumos o entrada y salidas para el modelo AED, como se describe más adelante en la Tabla 1 . Para lo anterior se tomaron los rubros financieros anuales presentados a la cámara de comercio de Barranquilla por estas organizaciones en el 2017.

En esta investigación se integran las técnicas de análisis envolvente de datos, análisis discriminante y redes neuronales artificiales para evaluar y predecir la eficiencia de pequeñas empresas a partir de rubros financieros de 90 empresas exportadoras suministrados por la Cámara de Comercio de BarranquillaColombia. En la primera fase de la investigación, se aplicó el Análisis Envolvente de Datos (AED) mediante el software DEA Solver Pro, tomando como variables de entrada salida los rubros financieros que se presentan en la Tabla 1. 
Tabla 1: Variables de entrada y salida del modelo DEA establecido

\begin{tabular}{|c|c|}
\hline Inputs & Outputs \\
\hline Total activos, & Ventas netas, \\
Patrimonio, & Utilidad neta \\
$\begin{array}{c}\text { Total pasivo, } \\
\text { Gastos operacionales, Costos } \\
\text { de ventas }\end{array}$ & Utilidad operacional \\
\hline
\end{tabular}

Para lo cual se adoptó el método no paramétrico de optimización con enfoque en las variables de salida CCRO que mide rendimientos a escala constante (Charnes, Cooper y Rhodes- Output) para clasificar las empresas en eficientes y no eficientes. Con esto se busca analizar como las variables de entrada inciden en la mejora de las variables de salidas asociadas al estado financiero de pérdidas o ganancias de las empresas objeto de esta investigación, como son ventas netas, utilidad neta y utilidad operacional. En segunda fase, se aplicó el análisis discriminante para evaluar la validez de clasificación lograda en el análisis envolvente de datos y la capacidad discriminante de las variables del estudio. En el análisis se utilizó como variables independientes los rubros financieros considerados en esta investigación y como variable dependiente la clasificación de empresas eficientes y no eficientes obtenida del AED. Para lo anterior, se utilizó el método de inclusión por pasos con cálculo de probabilidades previas según tamaños de grupo proporcionado por el software SPSS Statistics 25. En la tercera fase, se pronosticó la pertenencia de empresas a las categorías de eficientes y no eficientes, mediante la aplicación de modelos de Red Neuronal Artificial (RNA), utilizando el software SPSS Statistics 25 en el que se identificó una red de doble capa con función de entrada la Tangente Hiperbólica y de salida la función Sigmoide. Para lo anterior En el diseño del modelo, se tomó una partición del $60 \%$ de las empresas para el entrenamiento de la red, un $20 \%$ para la muestra de prueba y un $20 \%$ para la muestra de reserva. Se tomaron como variables independientes no estandarizadas los rubros financieros indicados anteriormente y como variable dependiente la clasificación de empresas eficientes y no eficientes obtenida en el análisis envolvente de datos. En la Figura 1, se ilustra las etapas del proceso. Con el propósito de validar el modelo de red neuronal seleccionado para el proceso de pronóstico, se desarrolló un análisis de validación cruzada compuesto por 10 particiones con 9 observaciones cada una. Con lo anterior, se hicieron 5 corridas del modelo de RNA.

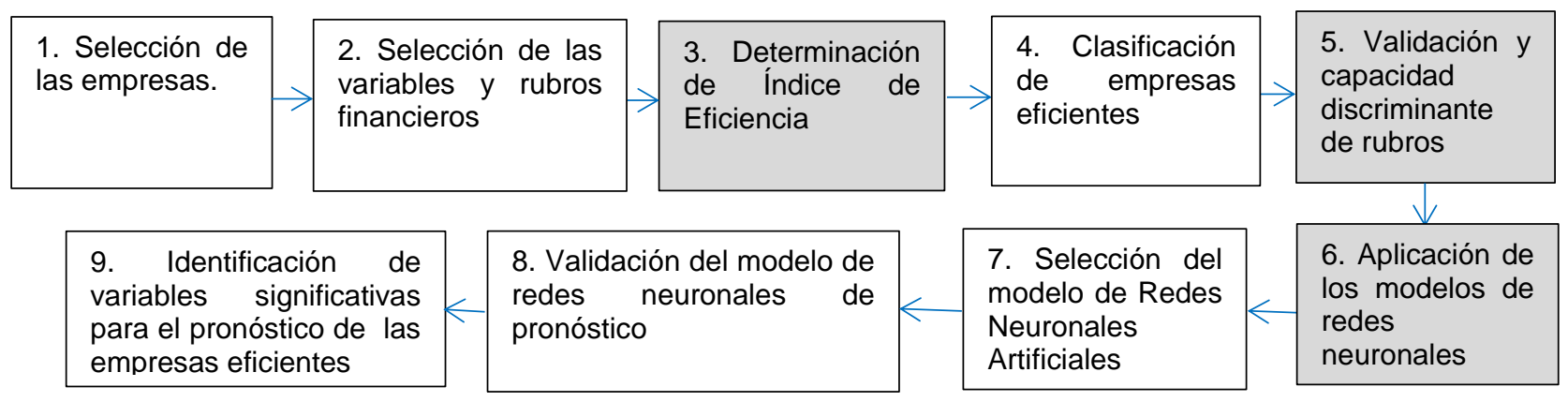

Fig. 1: Representación esquemática de la metodología propuesta

\section{RESULTADOS Y DISCUSIÓN}

Después de organizar la información y rubros asociados al modelo AED propuesto, se calcularon las eficiencias de las empresas objeto de estudio. Los resultados del índice de eficiencia, muestran que 79 empresas del sector no son eficientes en sus resultados de Ventas netas IP4, Utilidad neta IP7 y Utilidad operacional IP8 a partir de los recursos utilizados Total activos IP1, Patrimonio IP2, Total pasivo IP3, Ventas netas IP4, Gastos operacionales IP5, Costos de ventas IP6, Utilidad Neta IP7 y Utilidad operacional IP8. Las 11 empresas restantes, optimizan sus resultados al utilizar eficientemente los recursos evaluados, lo que las convierte en referentes para procesos de mejora del sector estudiado. En general, las 90 empresas analizadas mostraron un nivel de eficiencia técnica promedio del $41,38 \%$, con una desviación estándar de 0.3037. A continuación, en la Tabla 2 se presenta el Ranking de eficiencia de las pequeñas empresas exportadoras de Barranquilla, en donde se observa la puntuación de eficiencia obtenida para cada una de las 90 empresas estudiadas. La técnica asigna puntuación de 1 a las 11 empresas que mostraron ser eficientes. Se puede afirmar que de las 90 empresas estudiadas las primeras 11 DMU son eficientes todas ves que están en la frontera de la eficiencia y no presentan holguras o sus holguras son igual a cero. El resto de empresas que presentan holguras se consideran ineficientes. 
Tabla 2: Ranking de eficiencia de las pequeñas empresas exportadoras de Barranquilla

\begin{tabular}{|c|c|c|c|c|c|c|c|c|c|c|c|c|c|c|}
\hline Rank & DMU & P/tos & Rank & DMU & P/tos & & Rank & DMU & P/tos & Rank & DMU & P/tos \\
\hline 1 & 1 & 1 & 24 & 24 & 0,537 & & 47 & 47 & 0,327 & 70 & 70 & 0,179 \\
\hline 1 & 2 & 1 & 25 & 25 & 0,528 & 48 & 48 & 0,299 & 71 & 71 & 0,172 \\
\hline 1 & 3 & 1 & 26 & 26 & 0,521 & & 49 & 49 & 0,287 & 72 & 72 & 0,162 \\
\hline 1 & 4 & 1 & 27 & 27 & 0,518 & 50 & 50 & 0,287 & 73 & 73 & 0,144 \\
\hline 1 & 5 & 1 & 28 & 28 & 0,501 & & 51 & 51 & 0,284 & 74 & 74 & 0,120 \\
\hline 1 & 6 & 1 & 29 & 29 & 0,483 & 52 & 52 & 0,282 & 75 & 75 & 0,118 \\
\hline 1 & 7 & 1 & 30 & 30 & 0,477 & & 53 & 53 & 0,281 & 76 & 76 & 0,114 \\
\hline 1 & 8 & 1 & 31 & 31 & 0,446 & 54 & 54 & 0,274 & 77 & 77 & 0,100 \\
\hline 1 & 9 & 1 & 32 & 32 & 0,443 & & 55 & 55 & 0,271 & 78 & 78 & 0,098 \\
\hline 1 & 10 & 1 & 33 & 33 & 0,427 & 56 & 56 & 0,268 & 79 & 79 & 0,088 \\
\hline 1 & 11 & 1 & 34 & 34 & 0,404 & & 57 & 57 & 0,267 & 80 & 80 & 0,082 \\
\hline 12 & 12 & 0,990 & 35 & 35 & 0,399 & 58 & 58 & 0,261 & 81 & 81 & 0,078 \\
\hline 13 & 13 & 0,892 & 36 & 36 & 0,396 & & 59 & 59 & 0,257 & 82 & 82 & 0,077 \\
\hline 14 & 14 & 0,857 & 37 & 37 & 0,393 & 60 & 60 & 0,244 & 83 & 83 & 0,071 \\
\hline 15 & 15 & 0,823 & 38 & 38 & 0,388 & & 61 & 61 & 0,239 & 84 & 84 & 0,057 \\
\hline 16 & 16 & 0,810 & 39 & 39 & 0,382 & 62 & 62 & 0,233 & 85 & 85 & 0,050 \\
\hline 17 & 17 & 0,795 & 40 & 40 & 0,369 & & 63 & 63 & 0,219 & 86 & 86 & 0,040 \\
\hline 18 & 18 & 0,704 & 41 & 41 & 0,354 & 64 & 64 & 0,217 & 87 & 87 & 0,039 \\
\hline 19 & 19 & 0,676 & 42 & 42 & 0,354 & 65 & 65 & 0,216 & 88 & 88 & 0,023 \\
\hline 20 & 20 & 0,623 & 43 & 43 & 0,349 & 66 & 66 & 0,206 & 89 & 89 & 0,018 \\
\hline 21 & 21 & 0,597 & 44 & 44 & 0,338 & 67 & 67 & 0,205 & 90 & 90 & 0,017 \\
\hline 22 & 22 & 0,585 & 45 & 45 & 0,334 & 68 & 68 & 0,188 & & & \\
\hline 23 & 23 & 0,566 & 46 & 46 & 0,328 & 69 & 69 & 0,186 & & & \\
\hline
\end{tabular}

Con los resultados de clasificación de las empresas en los grupos eficientes y no eficientes, se realizó un análisis discriminante para evaluar el contraste de los grupos. La Tabla 3 se muestra los resultados del análisis de clasificación correcta del modelo con $54,5 \%$ para las empresas eficientes y del $91,1 \%$ para las empresas no eficientes con lo cual se logró un $72,8 \%$ de clasificación correcta de los casos agrupados originales. Se resalta que el mayor nivel de error se encuentra en la clasificación de la muestra de empresas eficientes $(45,5 \%)$, lo cual se explica en el bajo número de estas, en la conformación de la muestra. Por el contrario, en el grupo de empresas no eficientes, el nivel de error de clasificación fue de $8,9 \%$, lo que muestra el nivel de homogeneidad de las variables y por tanto su capacidad para discriminar empresas eficientes y no eficientes. Lo anterior se confirma con los resultados de la Tabla 4 concluyendo que la función discrimina bien la variable dependiente.

Tabla 3: Resultados de clasificación

\begin{tabular}{|c|c|c|c|c|c|}
\hline & \multirow{2}{*}{ Clasificación } & \multicolumn{2}{|c|}{ Pertenencia a grupos pronosticada } & \multirow{2}{*}{ Total } \\
\hline & & & Eficientes & No eficientes & \\
\hline \multirow{4}{*}{ Original } & \multirow{2}{*}{ Recuento } & Eficientes & 6 & 5 & 11 \\
\hline & & No eficientes & 7 & 72 & 79 \\
\hline & \multirow{2}{*}{$\%$} & Eficientes & 54,5 & 45,5 & 100,0 \\
\hline & & No eficientes & 8,9 & 91,1 & 100,0 \\
\hline
\end{tabular}

Tabla 4: Lambda de Wilks

\begin{tabular}{|c|c|c|c|c|}
\hline Prueba de funciones & $\begin{array}{c}\text { Lambda de } \\
\text { Wilks }\end{array}$ & Chi-cuadrado & gl & Sig. \\
\hline 1 &, 765 & 23,202 & 3 &, 000 \\
\hline
\end{tabular}

En las ecuaciones 5 y 6 , se presentan las funciones discriminantes lineales de Fisher para los grupos de eficiencia, a partir del cual se puede pronosticar la pertenencia de empresas en los grupos analizados.

\footnotetext{
$Z_{\text {eficientes }}=3,185 \mathrm{E}-9 * I P 1-4,046 \mathrm{E}-10 *$ IP2 $-3,310 \mathrm{E}-9 *$ IP3 $+1,236 \mathrm{E}-9 *$ IP $4-2,713 \mathrm{E}-9 *$ IP5 -

$1,233 \mathrm{E}-9 *$ IP6 + 7,131E $-9 *$ IP7 + 2,155E $-9 *$ IP8 $-3,026$

$Z_{\text {no eficientes }}=1,845 \mathrm{E}-9 * I P 1-1,254 \mathrm{E}-10 *$ IP2 $-9,402 \mathrm{E}-11 *$ IP3 $-4,602 \mathrm{E}-10 *$ IP4 $+1,563 \mathrm{E}-9 *$ IP5 +

$6,041 \mathrm{E}-10 *$ IP $-4,171 \mathrm{E}-11 *$ IP7 $-2,474 \mathrm{E}-10 *$ IP8 $-2,311$
} 
Verificación de supuestos: Según Lachenbruch (1975) el análisis discriminante no es particularmente sensible a las violaciones de menor importancia de la hipótesis de normalidad. Tabachnick y Fidell (2013), hacen algunas precisiones acerca de la robustez de esta técnica en relación con el tamaño de las muestras, sugiere un tamaño de muestra mayor a 20 para que el modelo sea robusto ante la violación del supuesto de multinormalidad. Para esta investigación se utilizaron 90 empresas como muestra. En relación a la homogeneidad de matrices de varianza-covarianza, los resultados de la prueba $\mathrm{M}$ de Box (Ver Tabla 5), permiten inferir que no existen diferencias entre las matrices de covarianza.

Con el propósito de excluir aquellas variables con correlación múltiple alta, se adoptó el método por etapas o paso a paso para la construcción del modelo discriminante. Los supuestos de linealidad y multicolinealidad y singularidad no son analizados, dado que para la construcción del modelo discriminante, se utilizó el método por etapas o paso a paso, teniendo en cuenta el criterio de tolerancia para seleccionar las variables que son incluidas. De este modo, aquellas variables que presentan una correlación múltiple elevada con las restantes variables arrojarán una baja tolerancia y no serían consideradas de cara a la construcción de la función discriminante (Rodríguez y Moreno; 2011).

Tabla 5: Resultados de la prueba de Box.

\begin{tabular}{|l|l|r|}
\hline \multicolumn{2}{|c|}{$M$ de Box } & \multicolumn{1}{c|}{41,541} \\
\hline \multirow{4}{*}{ F } & Aprox. & 6,138 \\
\cline { 2 - 3 } & gl1 & 6 \\
\cline { 2 - 3 } & gl2 & 1722,347 \\
\cline { 2 - 3 } & Sig. &, 000 \\
\hline
\end{tabular}

Es importante señalar que otras investigaciones han mostrado la pertinencia del Análisis discriminante para el análisis y la toma de decisiones en el estudio de sectores empresariales similares (Fontalvo, 2014).

Modelo de redes neuronales: En la construcción del modelo, se utilizó una red neuronal,con arquitectura del tipo perceptrón multicapa de dos capas ocultas con función de activación Tangente hiperbólica y función de activación de la capa de salida Sigmoide. Para el aprendizaje de la red, se utilizaron 57 muestras de entrenamiento, 15 muestras de prueba y 18 muestras de reserva. En el análisis las variables se estandarizaron y se utilizó el software IBM SPSS Statistics 25. En la Figura 2 se muestra la estructura del modelo RNA diseñado.

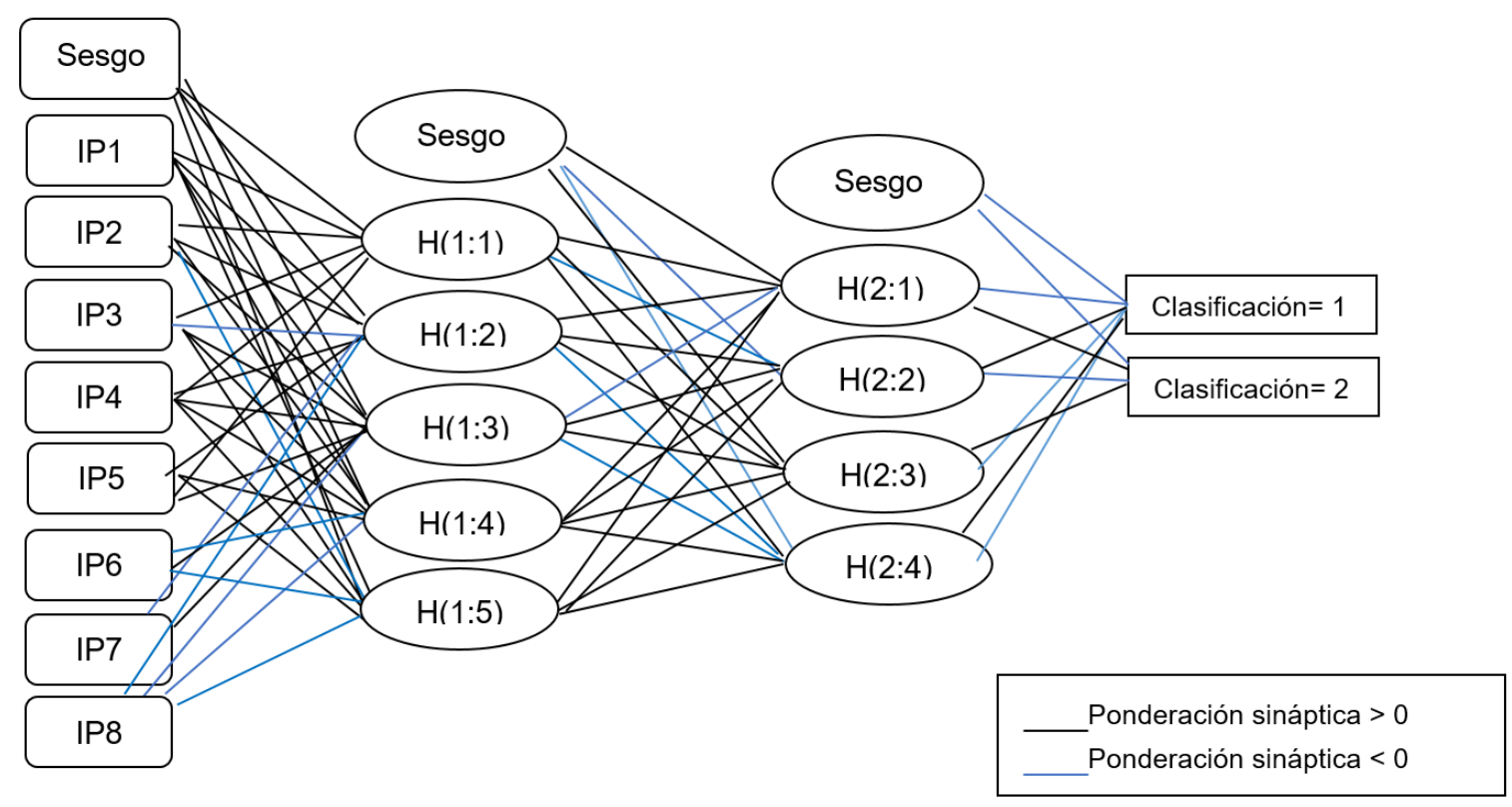

Fig. 2: Red neuronal para el pronóstico de pertenencia de empresas eficientes 
El modelo seleccionado generó una precisión para pronosticar la pertenencia de las pequeñas empresas exportadoras del $100 \%$ en la muestra de reserva, lo que muestra la alta consistencia del modelo DEA utilizado en la discriminación de empresas eficientes y no eficientes. En la Tabla 6, se muestran los resultados de pronóstico de la red RNA en las muestras de entrenamiento, prueba y reserva. Donde la variable dependiente o de clasificación se expresa por las empresas eficientes y no eficientes.

Tabla 6: Pronóstico de pertenencia de las empresas eficientes y no eficientes

\begin{tabular}{|l|c|c|c|c|}
\hline \multirow{3}{*}{ Muestra } & \multirow{2}{*}{ Observado } & \multicolumn{3}{|c|}{ Pronosticado } \\
\cline { 3 - 5 } & & Eficientes & No eficientes & Porcentaje correcto \\
\hline \multirow{3}{*}{ Entrenamiento } & Eficientes & 3 & 3 & $50,0 \%$ \\
\cline { 2 - 5 } & No eficientes & 0 & 51 & $100,0 \%$ \\
\cline { 2 - 5 } & Porcentaje global & $5,3 \%$ & $94,7 \%$ & $94,7 \%$ \\
\hline \multirow{3}{*}{ Pruebas } & Eficientes & 2 & 2 & $50,0 \%$ \\
\cline { 2 - 5 } & No eficientes & 0 & 11 & $100,0 \%$ \\
\cline { 2 - 5 } & Porcentaje global & $13,3 \%$ & $86,7 \%$ & $86,7 \%$ \\
\hline \multirow{3}{*}{ Reserva } & Eficientes & 1 & 0 & $100,0 \%$ \\
\cline { 2 - 5 } & No eficientes & 0 & 17 & $100,0 \%$ \\
\cline { 2 - 5 } & Porcentaje global & $5,6 \%$ & $94,4 \%$ & $100,0 \%$ \\
\hline
\end{tabular}

Validación del modelo de redes neuronales: En la Tabla 7, se muestran los resultados promedio de pronóstico en cada una de las muestras de entrenamiento, Prueba y Reserva, lográndose un 95,18\%, 88,89\% y 94,44\% de promedio porcentaje correcto de clasificación con lo cual se muestra la validez y relevancia del modelo de RNA. Cabe señalar que otras investigaciones muestran la pertinencia de clasificar grupos empresariales utilizando técnicas de cálculo multivariado (Fontalvo, 2012, Fontalvo, et al, 2011).

Tabla 7: Porcentajes de clasificación correcta del modelo RNA en las corridas por validación cruzada

\begin{tabular}{|c|c|c|c|c|c|c|c|}
\hline \multirow[b]{2}{*}{ Muestra } & \multirow[b]{2}{*}{ Observado } & \multicolumn{6}{|c|}{ Partición } \\
\hline & & 1 & 2 & 3 & 4 & 5 & $\begin{array}{c}\text { Promedio } \\
\text { porcentaje } \\
\text { correcto }\end{array}$ \\
\hline \multirow{3}{*}{ Entrenamiento } & Eficientes & 66,67 & 80,00 & 80,00 & 100,00 & 80,00 & 81,33 \\
\hline & No eficientes & 100,00 & 95,92 & 95,92 & 96,00 & 95,92 & 96,75 \\
\hline & Porcentaje global & 96,30 & 94,44 & 94,44 & 96,30 & 94,44 & 95,18 \\
\hline \multirow{3}{*}{ Prueba } & Eficientes & 50,00 & 100,00 & 75,00 & 75,00 & 66,67 & 73,33 \\
\hline & No eficientes & 100,00 & 93,33 & 92,86 & 92,86 & 86,67 & 93,14 \\
\hline & Porcentaje global & 88,89 & 94,44 & 88,89 & 88,89 & 83,33 & 88,89 \\
\hline \multirow{3}{*}{ Reserva } & Eficientes & 100,00 & 100,00 & 50,00 & 100,00 & 100,00 & 90,00 \\
\hline & No eficientes & 100,00 & 100,00 & 100,00 & 86,67 & 86,67 & 94,67 \\
\hline & Porcentaje global & 100,00 & 100,00 & 94,44 & 88,89 & 88,89 & 94,44 \\
\hline
\end{tabular}

En general, se resalta la capacidad de clasificación correcta del modelo RNA ( $94,44 \%$ en la muestra de reserva), en contraste con los resultados obtenidos en la técnica de análisis discriminante donde se alcanzó un $72,8 \%$.

Seguidamente, con el modelo de RNA, se realizó un análisis de importancia de los rubros para pronosticar la eficiencia de las empresas identificándose la Utilidad neta IP7, Utilidad operacional IP8 y Total patrimonio IP2 como los rubros con mayor significancia para discriminar las empresas en los grupos de eficiencia. Los resultados muestran la importancia de gestión de la utilidad y el patrimonio de las pequeñas empresas exportadoras para alcanzar mejores condiciones de eficiencia que conlleven a estas organizaciones a ser más competitivas en el contexto de los mercados en que se desarrollan. En la Tabla 8, se muestra el nivel de importancia de los rubros financieros para discriminar las empresas en eficientes y no eficientes en el modelo de RNA propuesto. 
Tabla 8: Importancia de los rubros financieros de las empresas exportadoras

\begin{tabular}{|l|c|c|}
\hline \multicolumn{1}{|c|}{ Rubros Financieros } & Importancia & Importancia normalizada (\%) \\
\hline Total activos & 0,041 & 11,9 \\
\hline Patrimonio & 0,199 & 57,7 \\
\hline Total pasivo & 0,062 & 18,0 \\
\hline Ventas netas & 0,039 & 11,3 \\
\hline Gastos operacionales & 0,063 & 18,4 \\
\hline Costos de ventas & 0,051 & 14,7 \\
\hline Utilidad Neta & 0,345 & 100,0 \\
\hline Utilidad operacional & 0,199 & 57,6 \\
\hline
\end{tabular}

\section{CONCLUSIONES}

Como aporte significativo esta investigación aporta nuevo conocimiento resultado de la articulación del conocimiento generado al articular las técnicas Análisis Envolvente de Datos, Análisis discriminante y Redes Neuronales artificiales que estructurado a través de un análisis racional y empírico aporta nuevo conocimiento en el sector exportador a nivel local, nacional e internacional. De lo anterior se propuso un método para evaluar y predecir la eficiencia técnica de las pequeñas empresas exportadoras de la ciudad de Barranquilla. El método propuesto es replicable en cualquier contexto nacional o internacional lo que constituye un aporte significativo a la comunidad científica y empresarial. Toda vez que aporta no solo criterios de evaluación de la eficiencia financiera, sino que además se establecieron criterios y un modelo de red neuronal artificial de doble capa para la predicción empresarial de nuevas empresas eficientes y no eficientes.

Adicionalmente, de la información empírica generada por la red neuronal artificial propuesta, se evidencia empíricamente que esta investigación aporta una estructura robusta para predecir el comportamiento de la eficiencia financiera en el sector objeto de esta investigación al observarse $94.44 \%$ de confiabilidad en la predicción global de la reserva. Lo que permite a los responsables de la toma de decisiones evaluar y predecir si una organización externa es eficiente o no con un grado de precisión alto. Así mimo, el método propuesto complementa trabajos como los desarrollados por Rodrigres y Rodrigues (2017), De Bock (2017), Kovacova y Kliestik (2017), Peres y Antao (2017), Du Jardin (2017), en los que se proponen metodologías y modelo predictivos empresariales

\section{REFERENCIAS}

Ahn, H., L. Neumann y N. Novoa, Measuring the Relative Balance of DMUs, European Journal of Operational Research, $221(2), 417-423$ (2012)

Alaka, H., L. Oyedele y otros cuatro autores, Systematic Review of Bankruptcy Prediction Models: Towards a Framework for Tool Selection, Expert Systems with Applications, 94, 164-184 (2017)

Caicedo, E. y J. López, Una Aproximación Práctica a las Redes Neuronales Artificiales, 1ª Ed., Programa Editorial Univeridad del Valle, Cali, Colombia (2009)

Cano, J., E. Campo y J. Baena, Application of DEA in International Market Selection for the Export of Goods, Revista Dyna, 84 (200), 376-382 (2017)

De Bock, K., The Best of Two Worlds: Balancing Model Strength and Comprehensibility in Business Failure Prediction Using Spline-Rule Ensembles, Expert Systems with Applications, 90, 23-39 (2017)

Delfín, O. y A. Melo, Eficiencia del Transporte Público en La Ciudad de Morelia, Michoacán (México) en el año 2015: Un análisis de la envolvente de datos, Revista Facultad de Ciencias Económicas: Investigación y Reflexión, 25 (2), 7$23(2017)$

Du Jardin, P., Dynamics of Firm Financial Evolution and Bankruptcy Prediction, Expert Systems with Applications, 75, 2543 (2017)

Fontalvo, T., Evaluación de la Gestión Financiera: Empresas del Sector Automotriz y Actividades Conexas En El Atlántico, Dimensión Empresarial, 10(2), 11-20 (2012)

Fontalvo, T., J. Morelos y E. De la Hoz, Aplicación del Análisis Discriminante para Evaluar el Mejoramiento de los Indicadores Financieros en las Empresas del Sector Extracción de Petróleo Crudo y Gas Natural en Colombia, Revista Soluciones de Postgrado EIA, 1(7), 11-26 (2011)

Fontalvo, T., A. Mendoza y D. Vishal, Comparative analysis of financial efficiency: a case study of BASC sector in Barranquilla, Prospectiva, 2(13), 16-24 (2015) 
Fontalvo, T., Aplicación de análisis discriminante para evaluar la productividad como resultado de la certificación BASC en las empresas de la ciudad de Cartagena, Contaduría y administración, 59 (1), 43-62 (2014)

García, A., El Análisis Envolvente de Datos, Herramienta para a Medición de la Eficiencia en Instituciones Sanitarias, Potencialidades y Limitaciones, Revista Cubana de Higiene y Epidemiología, 47(2) (2009)

Guzmán, I., Predicción de resultados empresariales versus medidas no paramétricas de eficiencia técnica: evidencia para pymes de la Región de Murcia, Presentado en VII Reunión de Economía Mundial, Madrid, Abril, (2005)

Hanafizadeh, P., H. Reza, A. Emrouznejad y M. Derakhshan, Neural network DEA for measuring the efficiency of mutual funds, International Journal of Applied Decision Sciences, 7(3), 255-269 (2014)

Heidari, M., M. Omid y A. Akram, Using Nonparametric Analysis (DEA) for Measuring Technical Efficiency in Poultry Farms, Revista Brasileira de Ciência Avícola, 13(4), 271-277 (2011)

Hornik, K., M. Stinchcombe y H. White, Multilayer Feedforward Networks are Universal Approximators, Neural Netw, 2 , 359-366 (1989)

Jiahe, A., X. Jiang y otros tres autores, Artificial Neural Network Prediction of the Microstructure of Rod Based on its Controlled Rolling and Cooling Process Parameters. Materials Science and Engineering, 344(1), 318-322 (2003)

Khalili-Damghani, K. y M. Mohammad-Taghavifard, Un enfoque difuso DEA de tres etapas para medir el rendimiento de un proceso en serie que incluye prácticas de JAT, índices de agilidad y objetivos en las cadenas de suministro. En t. J. of Services and Operations Management, 13(2), 147 - 188 (2012)

Kovacova, M. y T. Kliestik, Logit and Probit Application for the Prediction of Bankruptcy in Slovak Companies, EquilibriumQuarterly Journal of Economics and Economic Policy, 12(4), 775-791 (2017)

Lachenbruch, P., Discriminant Analysis, $1^{\text {a }}$ Ed., 1-128, Editorial Macmillan Pub Co., New York, EE.UU (1975)

Lins, M., M. Lobo y otros tres autores, Uso da Análise Envoltória de Dados (DEA) para Avaliação de Hospitais Universitários Brasileiros, Ciência y Saúde Coletiva, 12(4), 985-998 (2007)

Martín, O., M. Lopez y F. Martín, Artificial Neural Networks for Quality Control by Ultrasonic Testing In Resistance Spot Welding, Process. Techno, 183, 226-233 (2007)

McMillan, G., Process/Industrial Instruments and Controls Handbook, Mc.Graw Hill, México D.F., México (2013)

Moreno, J., O. López y J. Díaz, Productividad, Eficiencia y sus Factores Explicativos en el Sector de la Construcción en Colombia 2005-2010, Cuadernos de Economía, 33(63), 569-588 (2014)

Mojtaba, G., Efficiency improvement and resource estimation: a tradeoff analysis, https://doi.org/10.1504//JPQM.2018.094758, International Journal of Productivity and Quality Management, 25(2) (2018)

Peres, C. y M. Antao, The Use of Multivariate Discriminant Analysis to Predict Corporate Bankruptcy: A Review, Aestimatio-The leb International Journal of Finance, 14, 108-131 (2017)

Quesada, V., Estimación de la Eficiencia Mediante el Análisis Envolvente de Datos (DEA), Revista Panorama Económico, 11, 7-33 (2013)

Reddy, N., A. Rao, M. Chakraborty y B. Murty, Prediction of Grain Size of Al-7Si Alloy by Neural Networks, Engineering Science, 391,131-140 (2005)

Rodrigres, L. y L. Rodrigues, Economic-Financial Performance of the Brazilian Sugarcane Energy Industry: an Empirical Evaluation Using Financial Ratio, Cluster and Discriminant Analysis, Biomass and Bioenergy, 108, 289-296 (2017)

Rodríguez, J. y Á. Moreno, Fragilidad financiera de las firmas en Colombia, 2000 - 2006: Un análisis discriminante de un modelo minskiano, Documentos FCE ISSN: 2011-6322, 8(2), 1-42 (2011)

Senra, L., L. Nancil, J. Mello y L. Meza, Estudo sobre Métodos de Seleção de Variáveis em DEA. Pesquisa Operacional, 27(2), 191-207 (2007)

Soares, A., A. Pereira y S. Milagre, A Model For Multidimensional Efficiency Analysis of Public Hospital Management, Research on Biomedical Engineering, 33(4), 352-361 (2017)

Tabachnick, B. y L. Fidell, Using Multivariate Statistics, 6ª Ed., 34-48, Editorial Pearson, California, EE.UU (2013)

Tsay, H., H. Liu y J. Wu, Performance Assessment of Hong Kong Hotels. Journal of China Tourism Research, 13(2), 123140 (2017)

Velásquez, J., C. Franco y H. García, Un Modelo no Lineal para la Predicción de la Demanda Mensual de Electricidad en Colombia, Revista Estudios Gerenciales, 25(112), 37-54 (2009)

Visbal-Cadavid, D., A. Mendoza-Mendoza y K. Corredor-Carrascal, Evaluación del Desempeño Docente Mediante Análisis Envolvente de Datos: Un Estudio De Caso, Revista Entramado, 11(2), 218-225 (2015)

Zhou, Y., Z. Yan, N. Li y otros tres autores, Cloud-Data Envelopment Analysis Method Used for Assessment of Restoration Building Block Schemes, CSEE Journal of Power and Energy Systems, 1(2), 43-52 (2015) 
